\title{
An Interpolation Theorem for Quasimartingales in Noncommutative Symmetric Spaces
}

\author{
Congbian MA (D) and Guoxi Zhao \\ School of Mathematics and Information Science, Xinxiang University, Henan, China \\ Correspondence should be addressed to Guoxi Zhao; shuxinxi@xxu.edu.cn
}

Received 25 November 2020; Revised 7 January 2021; Accepted 18 January 2021; Published 31 January 2021

Academic Editor: Francesco Toppan

Copyright (c) 2021 Congbian MA and Guoxi Zhao. This is an open access article distributed under the Creative Commons Attribution License, which permits unrestricted use, distribution, and reproduction in any medium, provided the original work is properly cited.

Let $E$ be a separable symmetric space on $(0, \infty)$ and $E(\mathscr{M})$ the corresponding noncommutative space. In this paper, we introduce a kind of quasimartingale spaces which is like but bigger than $E(\mathscr{M})$ and obtain the following interpolation result: let $\widehat{E}(\mathscr{M})$ be the space of all bounded $E(\mathscr{M})$-quasimartingales and $1<p<p_{E}<q_{E}<q<\infty$. Then, there exists a symmetric space $F$ on $(0, \infty)$ with nontrivial Boyd indices such that $\widehat{E}(\mathscr{M})=\left(\widehat{L}_{p}(\mathscr{M}), \widehat{L}_{q}(\mathscr{M})_{F, K}\right.$.

\section{Introduction}

Let $E$ be a symmetric space on $(0, \infty)$ with the Fatou property and $1<p<p_{E}<q_{E}<q<\infty$. Kalton and MontgomerySmith [1] proved that there exists a symmetric space $F$ with nontrivial Boyd indices such that $E=\left(L_{p}((0, \infty)), L_{q}\right.$ $((0, \infty))_{F, K}$. As is now well-known, the preceding interpolation result can automatically lift to the noncommutative setting (see [[2], Theorem 3.4]): let $E$ be a symmetric space on $(0, \infty)$ with the Fatou property and $(\mathscr{M}, \tau)$ a semifinite von Neumann algebra. Then, the following are equivalent:

(i) $1<p<p_{E}<q_{E}<q<\infty$.

(ii) There exists a symmetric space $F$ on $(0, \infty)$ with nontrivial Boyd indices such that

$$
E(\mathscr{M})=\left(L_{p}(\mathscr{M}), L_{q}(\mathscr{M})\right)_{F, K} .
$$

In this paper, we replace the space $E(\mathscr{M})$ in (2) with a bigger and more complex space $\widehat{E}(\mathscr{M})$ (see Definition 3 ) and obtain a generalized interpolation result. Our main result can be stated as follows (see Section 2 for the unexplained notations).
Theorem 1. Let $E$ be a separable symmetric space on $(0, \infty)$ with $1<p<p_{E}<q_{E}<q<\infty$. Then, there exists a symmetric Banach function space $F$ on $(0, \infty)$ SSS with nontrivial Boyd indices such that

$$
\widehat{E}(\mathscr{M})=\left(\widehat{L}_{p}(\mathscr{M}), \widehat{L}_{q}(\mathscr{M})_{F, K}\right.
$$

\section{Preliminaries}

2.1. Noncommutative Spaces. Let $\mathscr{M}$ be a semifinite von Neumann algebra equipped with a faithful normal semifinite trace $\tau$. We denote by $L_{0}(\mathscr{M})$ the family of all $\tau$-measurable operators. Note that $\chi(\lambda, \infty)(|x|)$ is the spectral projection of $x \in L_{0}(\mathscr{M})$ associated with the interval $(\lambda, \infty)$. For $x \in L_{0}$ $(\mathscr{M})$, define its generalized singular number by

$$
\mu_{t}(x)=\inf \left\{\lambda>0: \tau\left(\chi_{(\lambda, \infty)}(|x|)\right) \leq t\right\}, t>0 .
$$

Note that the function $t \mapsto \mu_{t}(x)$ from $(0, \infty)$ into $[0, \infty)$ is right continuous and nonincreasing. For the case that $\mathscr{M}$ is the abelian von Neumann algebra $L(0, \infty)$ with the trace given by integration with respect to the Lebesgue measure, $L_{0}(\mathscr{M})$ is the space of all measurable functions, and $\mu(f)$ is 
the decreasing rearrangement of the measurable function $f$ (see $[3,4])$.

Recall that a Banach function space $\left(E,\|\cdot\|_{E}\right)$ on $(0, \infty)$ is called symmetric if for any $g \in E$ and any measurable function $f$ with $\mu(f) \leq \mu(g)$, we have $f \in E$ and $\|f\|_{E} \leq\|g\|_{E}$. The Köthe dual of $E$ is the function space defined by setting

$$
E^{\times}=\left\{f \in L_{0}(0, \infty): \int_{0}^{\infty}|f(t) g(t)| d t<\infty: \forall g \in E\right\} .
$$

When equipped with the norm $\|f\|_{E^{\times}}:=\sup \left\{\int_{0}^{\infty} \mid f(t)\right.$ $\left.g(t) \mid d t:\|g\|_{E} \leq 1\right\}, E^{\times}$is a symmetric Banach function space. For any $s>0$, we define the dilation operator $D_{s}$ on E by

$$
\left(D_{s} f\right)(t)=f(t / s), t>0, f \in E
$$

Define the lower and upper Boyd indices of $E$ by

$$
p_{E}:=\lim _{s \rightarrow \infty} \frac{\log s}{\log \left\|D_{s}\right\|} \text { and } q_{E}:=\lim _{s \rightarrow 0^{+}} \frac{\log s}{\log \left\|D_{s}\right\|} \text {, }
$$

respectively. It is well-known that $1 \leq p_{E} \leq q_{E} \leq \infty$, and we shall say that $E$ has nontrivial Boyd indices, whenever $1<$ $p_{E} \leq q_{E}<\infty$. We refer to $[1,5]$ for unexplained terminology from function space theory.

For a given symmetric Banach function space $\left(E,\|\cdot\|_{E}\right)$ on $(0, \infty)$, we define the corresponding noncommutative space by setting

$$
E(\mathscr{M}, \tau)=\left\{x \in L_{0}(\mathscr{M}): \mu_{t}(x) \in E\right\}
$$

equipped with the norm

$$
\|x\|_{E(\mathscr{M}, \tau)}:=\left\|\mu_{t}(x)\right\|_{E}
$$

It is well-known that $E(\mathscr{M}, \tau)$ is a Banach space and is referred to as the noncommutative symmetric space associated with $(\mathscr{M}, \tau)$ corresponding to the function space $\left(E,\|\cdot\|_{E}\right)$. Note that if $1 \leq p<\infty$ and $E=L_{p}(0, \infty)$, then $E(\mathscr{M}, \tau)=L_{p}(\mathscr{M}, \tau)$ is the usual noncommutative $L_{p}$-space associated with $(\mathscr{M}, \tau)$.

Recall that $l \infty$ is a von Neumann algebra equipped with the trace: $\gamma(a)=\sum a_{n}, a=\left(a_{n}\right) \in l_{\infty}^{+}$(see [6]). Now, let $\mathcal{N}=$ $\mathscr{M} \bar{\otimes} l_{\infty}$ be the von Neumann algebra tensor product and $v$ $=\tau \otimes \gamma$ the tensor trace. This gives rise to noncommutative spaces $E\left(\mathscr{M} \bar{\otimes} l_{\infty}\right)$. Note that $L_{p}\left(\mathscr{M} \bar{\otimes} l_{\infty}\right)$ coincides with the space $l_{p}\left(L_{p}(\mathscr{M})\right)$.

2.2. Noncommutative Martingales. A noncommutative probability space is a couple $(\mathscr{M}, \tau)$, where $\mathscr{M}$ is a finite von Neumann algebra and $\tau$ is a normal faithful trace with $\tau(1)=1$. Let $\left(\mathscr{M}_{n}\right)_{n \geq 1}$ be an increasing sequence of von Neumann subalgebras of $\mathscr{M}$ such that the union of the $\mathscr{M}_{n}{ }^{\prime} s$ is weak ${ }^{*}$ dense in $\mathscr{M}$. Let $\varepsilon_{n}$ be the conditional expectation with respect to $\mathscr{M}_{n}$.
Definition 2. A sequence $x=\left(x_{n}\right)_{n \geq 1}$ in $L_{1}(\mathscr{M})$ is called a sequence of martingale differences if $x_{n} \in E\left(\mathscr{M}_{n}\right)$ for $n \geq 1$ and if $\varepsilon_{n}\left(x_{n+1}\right)=0$ for all $n \geq 0$.

In this paper, we always consider noncommutative martingales associated with a noncommutative probability space unless explicit explanation.

2.3. Interpolation. Let $\left(X_{0}, X_{1}\right)$ be a compatible couple of quasi-Banach space. Its $K$-functional is defined by

$$
\begin{aligned}
& K_{t}\left(x ; X_{0}, X_{1}\right) \\
& \quad=\inf \left\{\left\|x_{0}\right\|_{X_{O}}+t\left\|x_{1}\right\|_{x_{1}}: x=x_{0}+x_{1}, x_{0} \in X_{0}, x_{1} \in X_{1}\right\},
\end{aligned}
$$

for $x \in X_{0}+X_{1}$ and $t>0$. Let $E$ be a symmetric Banach space on $(0, \infty)$. Set

$$
\|x\|_{\left(X_{0}, X_{1}\right)_{E, K}}=\left\|\frac{K_{t}\left(x ; X_{0}, X_{1}\right)}{t}\right\|_{E} .
$$

Then, the interpolation space $\left(X_{0}, X_{1}\right)_{E, K}$ is defined as $\left(X_{0}, X_{1}\right)_{E, K}=\left\{x \in X_{0}+X_{1}:\|x\|_{\left(X_{0}, X_{1}\right)_{E, K}}<\infty\right\}$ equipped with the norm $\|\cdot\|_{\left(X_{0}, X_{1}\right)_{E, K}}$.

\section{Main Result}

The main result in this section is Theorem 1, which extends the result of Kalton and Montgomery-Smith [1] to a $E(\mathscr{M})$ -quasimartingale spaces. We first introduce the quasimartingale spaces.

Definition 3. Let $E$ be a symmetric Banach function space on $(0, \infty)$. A sequence $x=\left(x_{n}\right)_{n \geq 1}$ is called a $E(\mathscr{M})$-quasimartingale with respect to $\left(\mathscr{M}_{n}\right)_{n \geq 1}$ if $x_{n} \in E\left(\mathscr{M}_{n}\right)$ for $n \geq 1$ and (with $\varepsilon_{0}=0, x_{0}=0$ )

$$
\left\|\sum_{n=1}^{\infty} \varepsilon_{n-1}\left(d x_{n}\right) \otimes e_{n}\right\| E\left(\mathscr{M} \bar{\otimes} l_{\infty}\right)<\infty .
$$

We set

$$
\|x\|_{\widehat{E}(\mathscr{M})}:=\sup _{n}\left\|y_{n}\right\|_{E(\mathscr{M})}+\left\|\sum_{n=1}^{\infty} \varepsilon_{n-1}\left(d x_{n}\right) \otimes e_{n}\right\| E\left(\mathscr{M} \bar{\otimes} l_{\infty}\right),
$$

where $\gamma_{n}=\sum_{k=1}^{n}\left(d x_{k}-\varepsilon_{k-1}\left(d x_{k}\right)\right)$ and $\left(e_{n}\right) n \geq 1$ denote the standard basic sequence of $l_{\infty}$.

If $\|x\|_{\widehat{E}(\mathscr{M})}<\infty, x$ is called a bounded $E(\mathscr{M})$-quasimartingale. The quasimartingale space $\widehat{E}(\mathscr{M})$ is defined as the space of all bounded $\widehat{E}(\mathscr{M})$-quasimartingales, equipped with the norm $\|\cdot\|_{\widehat{E}(\mathscr{M})}$. 
Remark 4.

(i) In the case $E=L_{p}$ for $1 \leq p<\infty$, we have that $\widehat{E}(\mathscr{M})$ $=\widehat{L_{p}}(\mathscr{M})$ and

$$
\|x\|_{\widehat{L}_{p}(\mathscr{M})}:=\sup _{n}\left\|y_{n}\right\|_{L_{p}(\mathscr{M})}+\left(\sum_{n=1}^{\infty}\left\|\varepsilon_{n-1}\left(d x_{n}\right)\right\|_{L p(\mathscr{M})}^{p}\right)^{1 / p}
$$

(ii) Let $x=\left(x_{n}\right)_{n \geq 1}$ be a bounded $E(\mathscr{M})$-quasimartingale. Set $z_{n}=\sum_{k=1}^{n}\left(\mathscr{E}_{k-1}\left(d x_{k}\right)\right)$ and $y_{n}=x_{n}-z_{n}(n \geq 1)$. Then, $z=\left(z_{n}\right)_{n \geq 1}$ is a predicable $E(\mathscr{M})$-quasimartingale with $z_{1}=0$, and $y=\left(y_{n}\right)_{n \geq 1}$ is a bounded $E(\mathscr{M})$ -martingale. Moreover, the decomposition,

$$
x=y+z,
$$

is unique (see Lemma 2.5, [7]).

The following lemma is the key ingredient of our proof of Theorem 7.

Lemma 5. Let $E$ be a separable symmetric space on $(0, \infty)$ with $1<p_{E} \leq q_{E}<\infty$. Then,

$$
(E \wedge(\mathscr{M}))^{*}=E \wedge^{\times}(\mathscr{M})
$$

with equivalent norms.

Proof. Let $\mu=\left(\mu_{n}\right)_{n \geq 1} \in E \wedge^{\times}(\mathscr{M})$ and $x=\left(x_{n}\right)_{n \geq 1} \in \widehat{E}(\mathscr{M})$. Let $\mu_{n}=v_{n}+\omega_{n}$ and $x_{n}=y_{n}+z_{n}\left({ }_{n \geq 1}\right)$ be the decomposition of $\mu$ and $x$ as in (14). Then, $y=\left(y_{n}\right)_{n \geq 1}$ is a bounded $E(\mathscr{M})$-martingale and $v=\left(v_{n}\right)_{n \geq 1}$ is a bounded $\widehat{E}(\mathscr{M})$ -martingale. Thus, there exist $y_{\infty} \in E(\mathscr{M})$ and $\nu_{\infty} \in E^{\times}(\mathscr{M})$ such that

$$
y_{n} \stackrel{E(\mathscr{M})}{\longrightarrow} y \infty, v_{n} \stackrel{E^{\times}(\mathscr{M})}{\longrightarrow} v_{\infty} .
$$

Now, we define a linear functional on $\widehat{E}(\mathscr{M})$ by

$$
l_{\mu}(x)=\tau\left(v_{\infty} y_{\infty}\right)+\sum_{n=1}^{\infty} \tau\left(d \omega_{n} d z_{n}\right)
$$

Then, by Hölder's inequality,

$$
\begin{aligned}
\left|l_{\mu}(x)\right| \leq & \left\|v_{\infty}\right\|_{E^{\times}(\mathscr{M})}\left\|y_{\infty}\right\|_{E(\mathscr{M})} \\
& +\tau \otimes \gamma\left(\sum_{n=1}^{\infty} d \omega_{n} \otimes e_{n} \sum_{n=1}^{\infty} d z_{n} \otimes e_{n}\right) \\
\leq & \left\|v_{\infty}\right\|_{E^{\times}(\mathscr{M})}\left\|y_{\infty}\right\|_{E(\mathscr{M})} \\
& +\left\|\sum_{n=1}^{\infty} d \omega_{n} \otimes e_{n}\right\|\left\|_{E\left(\mathscr{M} \otimes l_{\infty}\right)}\right\| \sum_{n=1}^{\infty} d z_{n} \otimes e_{n} \| E^{\times}\left(\mathscr{M} \bar{\otimes} l_{\infty}\right) \\
\leq & \|\mu\|_{E \wedge^{\times}(\mathscr{M})}\|x\|_{\bar{E}(\mathscr{M})} .
\end{aligned}
$$

Thus, $l_{\mu}(x)$ is continuous on $\widehat{E}(\mathscr{M})$ and $\left\|l_{\mu}\right\| \leq\|\mu\|_{E \wedge^{\times}(\mathscr{M})}$.

We pass to the converse inclusion. Let $l \in(E \wedge(\mathscr{M}))^{*}$. Let $l_{1}$ be the restriction of $l$ on $E(\mathscr{M})$. Noting that $(E(\mathscr{M}))^{*}=$ $E^{\times}(\mathscr{M})$, there exists an operator $v \in E^{\times}(\mathscr{M})$ and $\|v\|_{E^{\times}(\mathscr{M})} \leq$ $\|l\|$ such that

$$
l_{1}(a)=\tau(a v), a \in E(\mathscr{M})
$$

On the other hand, let $F_{E}(M)$ be the space of all sequences $d b=\left(d b_{n}\right)_{n \geq 1}$ such that $b=\left(b_{n}\right)_{n \geq 1}$ is a predictable $E(\mathscr{M})$-quasimartingale with $b_{1}=0$ equipped with the norm $\|d b\|_{F_{E}(\mathscr{M})}=\left\|\sum_{n=1}^{\infty} d b_{n} \otimes e_{n}\right\| E(\mathscr{M} \bar{\otimes} l \infty)$. Define a functional on $F_{E}(\mathscr{M})$ by

$$
l_{2}(d b)=l(b), d b=\left(d b_{n}\right)_{n \geq 1} \in F_{E}(M) .
$$

Then, by the inequality

$$
\left|l_{2}(d b)\right| \leq\|l\|\|b\|_{\widehat{E}(\mathscr{M})}=\|l\|\|d b\|_{F_{E}(\mathscr{M})},
$$

we have $l_{2}$ is a continuous linear functional on $F_{E}(M)$ and $\left\|l_{2}\right\| \leq\|l\|$. Note that $F_{E}(M)$ is isometric to the subspace of $E(M \bar{\otimes} l \infty)$. By the Hahn-Banach theorem, $l_{2}$ extends to a functional on $E(M \bar{\otimes} l \infty)$. Since $(E(M \bar{\otimes} l \infty)) *=E \times$ $\left(M \bar{\otimes} l_{\infty}\right)$, the representation theorem allows us to find a sequence $\left(W_{n}^{\prime}\right)_{n \geq 1} \in E^{\times}\left(M \bar{\otimes} l_{\infty}\right)$ such that

$$
\begin{aligned}
l_{2}(s) & =\sum_{n=1}^{\infty} v\left(\left(w_{n}^{\prime} \otimes e_{n}\right)\left(s_{n} \otimes e_{n}\right)\right) \\
& =\sum_{n=1}^{\infty} \tau\left(w_{n}^{\prime} s_{n}\right),\left(s_{n}\right)_{n \geq 1} \in E\left(\mathscr{M} \bar{\otimes} l_{\infty}\right),
\end{aligned}
$$

and

$$
\left\|\sum_{n=1}^{\infty} w_{n}^{\prime} \otimes e_{n}\right\|_{E^{\times}\left(M \bar{\otimes} l_{\infty}\right)} \leq\left\|l_{2}\right\| .
$$

Set $\omega_{1}=0$ and $w_{n}=\sum_{k=1}^{n} \varepsilon_{k-1}\left(w_{k}^{\prime}\right)(n \geq 2)$. For any $d b=$ $\left(d b_{n}\right)_{n \geq 1} \in F_{E}(M)$, noting that $d b=\left(d b_{n}\right)_{n \geq 1}$ is predicable, it follows from (22) that 


$$
\begin{aligned}
l_{2}(d b) & =\sum_{n=1}^{\infty} \tau\left(\varepsilon_{n-1}\left(w_{n}^{\prime} d b_{n}\right)\right)=\sum_{n=1}^{\infty} \tau\left(d b_{n} \varepsilon_{n-1}\left(w_{n}^{\prime}\right)\right) \\
& =\sum_{n=1}^{\infty} \tau\left(d w_{n} d b_{n}\right) .
\end{aligned}
$$
and

It is easy to see that $\omega=\left(\omega_{n}\right)_{n \geq 1}$ is predictable with $\omega_{1}=0$

$$
\begin{aligned}
\left\|\sum_{n=1}^{\infty} d w_{n} \otimes e_{n}\right\|_{E^{\times}\left(\mathcal{M} \bar{\otimes} l_{\infty}\right)} & =\left\|\sum_{n=1}^{\infty} \varepsilon_{n-1}\left(w_{n}^{\prime}\right) \otimes e_{n}\right\|_{E^{\times}\left(\mathcal{M} \bar{\otimes} l_{\infty}\right)} \\
& \leq\left\|\sum_{n=1}^{\infty} w_{n}^{\prime} \otimes e_{n}\right\|_{E^{\times}\left(M \bar{\otimes} l_{\infty}\right)} \leq\left\|l_{2}\right\| .
\end{aligned}
$$

Set $\mu_{n}=v_{n}+\omega_{n}(n \geq 1)$, where $v_{n}=\varepsilon n(v)(n \geq 1)$. Then, $\mu=\left(\mu_{n}\right)_{n \geq 1} \in E \wedge^{\times}(M)$ and

$$
\|\mu\|_{E \wedge^{\times}(\mathscr{M})}=\|v\|_{E^{\times}(\mathscr{M})}+\left\|\sum_{n=1}^{\infty} d w_{n} \otimes e_{n}\right\|_{E^{\times}\left(\mathscr{M} \bar{\otimes} l_{\infty}\right)} \leq 2\|l\| .
$$

For any $x=\left(x_{n}\right)_{n \geq 1} \in \widehat{E}(\mathscr{M})$, let $x_{n}=y_{n}+z_{n}(n \geq 1)$ be its decomposition as in (14).

Noting that $y=\left(y_{n}\right)_{n \geq 1}$ is a bounded $E(\mathrm{M})$ martingale and $d z=\left(d z_{n}\right)_{n \geq 1} \in F_{E}(M)$, it follows from (19) and (24) that

$$
l(x)=l(y)+l(z)=\tau\left(y_{\infty} v_{\infty}\right)+\sum_{n=1}^{\infty} \tau\left(d w_{n} d z_{n}\right)
$$

The proof is completed.

The following lemma is about the duality theorem of interpolation spaces.

Lemma 6 (see [2]). Let $E$ be separable and $\left(X_{1}, X_{2}\right)$ be a couple of Banach spaces such that $X_{1} \cap X_{2}$ is dense in both $X_{1}$ and $X_{2}$. Then,

$$
\left(X_{1}, X_{2}\right)_{E, K}^{*}=\left(X_{2}^{*}, X_{1}^{*}\right)_{E^{\times}, K}
$$

Proof of Theorem 7. Let $x \in\left(\widehat{L}_{p}(\mathscr{M}), \widehat{L}_{q}(\mathscr{M})\right)_{F, K}$ and $x=x^{0}$ $+x^{1}$ be a decomposition of $x$, where $x^{0} \in L_{p}(\mathscr{M}), x^{1} \in L_{q}(\mathscr{M})$. Let $x_{n}^{k}=y_{n}^{k}+z_{n}^{k}(n \geq 1)$ be the decomposition of $x^{k}(k=0,1)$ as in (14). Then, $y^{0}$ is a bounded $L_{p}(M)$-martingale, and $y^{1}$ is a bounded $L_{q}(M)$-martingale. Thus, there exist $y_{\infty}^{0} \in L_{p}(\mathscr{M})$ and $y_{\infty}^{1} \in L_{q}(\mathscr{M})$ such that

$$
\begin{aligned}
y_{n}^{0} L_{p}(\mathscr{M}) & \rightarrow y_{\infty}^{0}, y_{n}^{1} L_{p}(\mathscr{M}) \rightarrow y_{\infty}^{1}, \\
\left\|y_{\infty}^{0}\right\|_{L_{p}(\mathscr{M})} & =\sup _{n}\left\|y_{n}^{0}\right\|_{L_{p}(\mathscr{M})},\left\|y_{\infty}^{1}\right\|_{L_{p}(\mathscr{M})} \\
& =\sup _{n}\left\|y_{n}^{1}\right\|_{L_{p}(\mathscr{M})} .
\end{aligned}
$$

Using Definition 3, we get that

$$
\begin{aligned}
\left\|x^{0}\right\|_{\widehat{L}_{p}(\mathscr{M})} & =\left\|y_{\infty}^{0}\right\|_{L_{p}(\mathscr{M})}+\left\|d z^{0}\right\|_{L_{p}\left(\mathscr{M} \bar{\nabla} l_{\infty}\right)}\left\|x^{1}\right\|_{\widehat{L}_{p}(\mathscr{M})} \\
& =\left\|y_{\infty}^{1}\right\|_{L_{q}(\mathscr{M})}+\left\|d z^{1}\right\|_{L_{q}\left(\mathscr{M} \bar{\nabla} l_{\infty}\right)} .
\end{aligned}
$$

Set $y=y_{\infty}^{0}+y_{\infty}^{1}, z=z^{0}+z^{1}$.Then, by the definition of $K$ -functionals and (30),

$$
\begin{aligned}
& K_{t}\left(y ; L_{p}(\mathscr{M}), L_{q}(\mathscr{M})\right)+K_{t}\left(d z ; L_{p}\left(\mathscr{M} \bar{\otimes} l_{\infty}\right), L_{q}\left(\mathscr{M} \bar{\otimes} l_{\infty}\right)\right) \\
& \leq \leq y_{\infty}^{0}\left\|_{L_{p}(\mathscr{M})}+t\right\| y_{\infty}^{1}\left\|_{L_{q}(\mathscr{M})}+\right\| d z^{0} \|_{L_{p}\left(\mathscr{M} \bar{\otimes} l_{\infty}\right)} \\
& \quad+t\left\|d z^{1}\right\|_{L_{q}\left(\mathscr{M} \bar{\otimes} l_{\infty}\right)}=\left\|x^{0}\right\|_{\hat{L}_{p}(\mathscr{M})}+t\left\|x^{1}\right\|_{\hat{L}_{q}(\mathscr{M})} .
\end{aligned}
$$

Thus, taking infimum over all decomposition of $x$, we obtain

$$
\begin{aligned}
& K_{t}\left(y ; L_{p}(M), L_{q}(M)\right)+K_{t}\left(d z ; L_{p}\left(M \bar{\otimes} l_{\infty}\right), L_{q}\left(M \bar{\otimes} l_{\infty}\right)\right) \\
& \quad \leq K_{t}\left(x ; \widehat{L}_{p}(M), \widehat{L}_{q}(M)\right) .
\end{aligned}
$$

Therefore, using the equality $\|x\|_{\left(X_{0}, X_{1}\right) F, K}=$ $\left\|K_{t}\left(x ; X_{0}, X_{1}\right) / t\right\|_{F}$, we have

$$
\begin{aligned}
& \|y\|_{\left(L_{p}(M), L_{q}(M)\right) F, K}+\|d z\|_{\left.L_{p}\left(M \bar{\otimes} l_{\infty}\right), L q\left(M \bar{\otimes} l_{\infty}\right)\right) F, K} \\
& \quad \leq 2\|x\|_{\left(\widehat{L}_{p}(M), \widehat{L}_{q}(M)\right) F, K} .
\end{aligned}
$$

It follows from the equality $E(M)=\left(L_{p}(M), L_{q}(M)\right)_{F, K}$ that

$$
\|x\|_{\widehat{E}(M)}=\|y\|_{E(M)}+\|d z\|_{E\left(M \bar{\otimes} l_{\infty}\right)} \leq 2\|x\|_{\left(\widehat{L}_{p}(M), \widehat{L}_{q}(M)\right)_{F, K}} .
$$

For any $x \in\left(\widehat{L}_{p}(M), \widehat{L}_{q}(M)\right)_{\mathrm{F}, \mathrm{K}^{\prime}}$, we obtain $x \in \widehat{E}(M)$ which implies that

$$
\widehat{E}(\mathscr{M}) \supset\left(\widehat{L}_{p}(\mathscr{M}), \widehat{L}_{q}(\mathscr{M})\right)_{F, K} .
$$

Similarly, we have

$$
E \wedge^{\times}(\mathscr{M}) \supset\left(\widehat{L}_{q^{\prime}}(\mathscr{M}), \widehat{L}_{p^{\prime}}(\mathscr{M})\right)_{F^{\times}, K},
$$

where $p^{\prime}$ and $q^{\prime}$ denote the conjugate index of $p$ and $q$. By Lemma 5 and Lemma 6, we obtain that 


$$
\begin{aligned}
\widehat{E}(\mathscr{M}) & =\left(E \wedge^{\times}(\mathscr{M})\right)^{*} \subset\left(\widehat{L}_{q^{\prime}}(\mathscr{M}), \widehat{L}_{p^{\prime}}(\mathscr{M})\right)_{F^{\times}, K}^{*} \\
& =\left(\widehat{L}_{p}(\mathscr{M}), \widehat{L}_{q}(\mathscr{M})\right)_{F, K} .
\end{aligned}
$$

Thus,

$$
\widehat{E}(\mathscr{M})=\left(\widehat{L}_{p}(\mathscr{M}), \widehat{L}_{q}(\mathscr{M})\right)_{F, K} \cdot
$$

The proof is completed.

\section{Data Availability}

The data used to support the findings of this study are available from the corresponding author upon request.

\section{Conflicts of Interest}

The authors declare that they have no conflicts of interest.

\section{Acknowledgments}

This work was supported by the National Natural Science Foundation of China $(11801489,11671308)$.

\section{References}

[1] N. Kalton and S. Montgomery-Smith, "Interpolation of Banach spaces," in Handbook of the geometry of Banach spaces, vol. 2, pp. 1131-1175, Elsevier.

[2] P. Dodds, T. Dodds, and B. de Pagter, "Fully symmetric operator spaces," Integral Equations and Operator Theory, vol. 15, no. 6, pp. 942-972, 1992.

[3] J. Lindenstrauss and L. Tzafriri, "Classical Banach spaces. II. Function spaces," in Ergebnisse der Mathematik und ihrer Grenzgebiete, vol. 97, Springer-Verlag, Berlin-New York, 1979.

[4] C. Ma and Y. Hou, "Interpolation of noncommutative quasimartingale spaces," Annals of Functional Analysis, vol. 7, no. 3, pp. 484-495, 2016.

[5] T. Bekjan and Z. Chen, "Interpolation and $\Phi$-moment inequalities of noncommutative martingales," Probability Theory and Related Fields, vol. 152, no. 1-2, pp. 179-206, 2012.

[6] Y. Jiao, F. Sukochev, D. Zanin, and D. Zhou, "Johnson-Schechtman inequalities for noncommutative martingales," Journal of Functional Analysis, vol. 272, pp. 979-1016, 2017.

[7] C. Ma and Y. Hou, "Quasi-martingale Inequalities in Noncommutative Symmetric Spaces," Bulletin of the Malaysian Mathematical Sciences Society, vol. 42, no. 5, pp. 2639-2655, 2018. 